\begin{tabular}{|c|c|c|}
\hline PORT SAID RESEARCH ENGINEERING JOURNAL & Faculty of Engineering - Port Said University \\
Volume 17 No. 1 pp: $42: 55$ & \\
\hline
\end{tabular}

\title{
PERFORMANCE OF SRTM 30 CREATED FROM SRTM 90
}

\author{
Ashraf Aly Elkoushy ${ }^{1}$
}

\section{ABSTRACT}

The main objective of this study was to evaluate the prediction of SRTM30 from the freely available SRTM90 by using means of different interpolation orders. In this study three sorts of data were used, actual data of SRTM30 of USA which were used as simulated data, SRTM90 data which were used as a real data whereas no idea bout SRTM30 are available and ground-truth data that were used for the verification of the predicted SRTM30. SRTM90 data used in this study was concerned with part area of Egypt extended from $29^{\circ} \mathrm{N}$ and $32^{\circ} \mathrm{E}$ to $30^{\circ} \mathrm{N}$ and $33^{\circ} \mathrm{E}$ which have all features of terrain surface. The ground truth data were used within the same study area of SRTM90 to examine and analyze the characteristics of the interpolation. The interpolation used in this study is polynomial with different types of orders. The results obtained indicated an improvement in the prediction of SRTM30. However, these results cannot be approved completely, whereas interpolation was just a foresight procedure, at least it was helpful to improve the terrain surface or DEM produced from SRTM90.

\section{INTRODUCTION}

The 30-meter and 90-meter SRTM data are without restraint and they are freely available for the USA by using the Seamless Data Distribution as reported by [19] and [20] and ftp://e0mss21u.ecs.nasa.gov/srtm or ftp://e0srp01u.ecs.nasa.gov/srtm/.

The data distributed via ftp from the Land Processes Distributed Active Archive Center (LP-DAAC) are different from its Seamless Data Distribution System (SDDS, 'Seamless Server') presented by [19] and [20]. The three arc-second sampled data is generated by (SDDS, 'Seamless Server') from the one arc-second called "sampling" where each three arc-second data point is generated by selecting the center sample of the $3 \times 3$ array of one arc-second points surrounding the post location. For the LP-DAAC, three arc second data, each point is the average of the nine one arc-second samples surrounding the post; "averaging".

SRTM data are organized into individual rasterized cells or tiles, each covering one degree by one degree in latitude and longitude. Sample spacing for individual data points is either 1 arc-second or 3 arc-seconds defined as SRTM1 and SRTM3, respectively. Since one arc-second at the equator corresponds to roughly 30 meters in horizontal extent, the SRTM1 and SRTM3 are sometimes referred to as "30 meter" or "90 meter" data. The 1 arc-second original data have been made available to the public only for North America.

SRTM land elevations data could be considered accurate whereas the data for sea areas show a lot of variation from the expected zero elevation.

${ }^{1}$ Civil Engineering Department, Faculty of Engineering- Port Said University, Port Said, Egypt, E-mail:dr.aelkoushy@gmail.com
Several studies evaluated the role of SRTM in improving the DEM resolution such as [8] where the authors presented a practical use of SRTM data in the tropics and compared it with digital elevation models generated from cartographic data. They found that SRTM three arc-second DEMs perform well for hydrological modeling.

The studied vertical accuracy of the SRTM and DTED level 1, and concluded that SRTM and DTED-1 were not typical of plane regions and sloping regions presented a greater mean error than the plane ones was presented by [12]. The steps necessary to improve the resolution of SRTM 30 using variograms modeling and kriging and compared his results with those obtained by regularized splines had been reported by [3].

A methodology for refinement $90 \mathrm{~m} \mathrm{DEM}$ and reconstructed SRTM30 from SRTM90 using bicubic polynomial interpolation at USA was presented by [9]. The authors found that using SRTM90 as a source to generate DEM was similar to the original SRTM30.

Evaluated the planimetric adjustment of elevation data to the SRTM Digital Elevation Model by using correlation which applied to geo-reference any geographic dataset was presented by [5]. A comparison between the decimation and averaging methods of DEM re-sampling was studied by [1] and found that decimated SRTM dataset was preferred because it provides the 'true' values of elevations.

\section{INTERPOLATION METHODS}

Interpolating of a DEM involves finding elevations and locations of new grid posts based on the posts and elevations of the input DEM. The determination of a surface can be defined by regularly spaced data that has 
been stated in literature as the points $\left(X_{i}, Y_{i}, Z_{i}\right)$, for $i=$ $1,2 \ldots n$, over some domain. A function $\mathrm{Z}=f(X, Y)$ is desired to reproduces the given points, and produces a reasonable estimate of the surface $(Z)$ at all other points $(\mathrm{X}, \mathrm{Y})$ in the given domain.

$$
\begin{aligned}
Z(x, y)=a_{0} & +a_{1} X+a_{2} Y+a_{3} X^{2}+a_{4} X Y+a_{5} Y^{2} \\
& +a_{6} X^{3}+a_{7} X^{2} Y+a_{8} X Y^{2}+a_{9} Y^{3} \\
& +a_{10} X^{4}+a_{11} X^{3} Y+a_{12} X^{2} Y^{2}+a_{13} X Y^{3} \\
& +a_{14} Y^{4}+\ldots \ldots \ldots+a_{m n} X^{m} Y^{n}
\end{aligned}
$$

The polynomial of equation (1) can be expanded to any desired degree, although there are computational limits because of rounding error. The unknown coefficients are found by using least squares technique and solving a set of simultaneous linear equations which include the sums of powers and cross products of the $\mathrm{X}, \mathrm{Y}$, and $\mathrm{Z}$ values. Once the coefficients have been estimated, the polynomial function can be evaluated at any point within the map area. It is a simple matter to create a grid matrix of values by substituting the coordinates of the grid nodes into the polynomial and calculating an estimate of the surface for each node.

This study was considered with different four degrees of polynomial outlined briefly below which could be considered enough to solve the problem of DEM prediction.

A first order linear trend surface equation which yields a plane trend surface has the form:

$$
Z(x, y)=a_{0}+a_{1} X+a_{2} Y
$$

For second-order polynomial trend surfaces, the general equation is:

$$
Z(x, y)=a_{0}+a_{1} X+a_{2} Y+a_{3} X^{2}+a_{4} X Y+a_{5} Y^{2}
$$

Third-order polynomial trend surfaces (bi-cubic method):

$$
\begin{gathered}
Z(x, y)=a_{0}+a_{1} X+a_{2} Y+a_{3} X^{2}+a_{4} X Y+a_{5} Y^{2} X^{2} Y \\
+a_{6} X^{3}+a_{7} a_{8} X Y^{2}+a_{9} Y^{3}
\end{gathered}
$$

Fourth-order polynomial trend surfaces which had 15 coefficients to be determined, which can be considered enough for surface prediction.

$$
\begin{aligned}
Z(x, y)=a_{0} & +a_{1} X+a_{2} Y+a_{3} X^{2}+a_{4} X Y+a_{5} Y^{2} \\
& +a_{6} X^{3}+a_{7} X^{2} Y+a_{8} X Y^{2}+a_{9} Y^{3}+a_{10} X^{4} \\
& +a_{11} X^{3} Y+a_{12} X^{2} Y^{2}+a_{13} X Y^{3}+a_{14} Y^{4}
\end{aligned}
$$

The previous interpolation with different orders polynomial degree was considered. Likewise, this study provided a guide to examine the most effective order that could predict of SRTM30 from SRTM90.

\section{INTERPOLATION PROCESS OF ACTUAL DATA}

The one arc second original data have been made available to the public only for North America
USA which have one arc-second, SRTM30. The area extends from $39^{\circ} \mathrm{N}$ to $42^{\circ} \mathrm{N}$ latitudes and $104^{\circ} \mathrm{W}$ to $107^{\circ} \mathrm{W}$ longitudes was considered and these data are freely available. This wide area covered three degree by three degree squared to cover the overlapped pixels while the study concerned only with the area between $40^{\circ} \mathrm{N}$ to $41^{\circ} \mathrm{N}$ latitudes and $105^{\circ} \mathrm{W}$ to $106^{\circ} \mathrm{W}$ as shown in Figure 1, which covered $9408 \mathrm{~km}^{2}$ (3601 rows* 3601columns). Moreover, one pixel surrounded this area was taken into consideration. Therefore, this area grows to cover $9418 \mathrm{~km}^{2}$ (3603 rows* 3603 columns) with pixel size 0.000278 arc degrees.

The selected area extended and contained flat terrain, smoothly sloped, sharply sloped, and rugged mountains as shown in Figure 1. It included elevations varied from 1466 to 4293 meter. SRTM30 was converted into SRTM90 by using two methods of averaging and sampling which were used in another study by the author. Thus, SRTM90 data were used as a simulated data, which have (1201 rows* 1201 columns) with pixel size 0.000833 arc degrees.
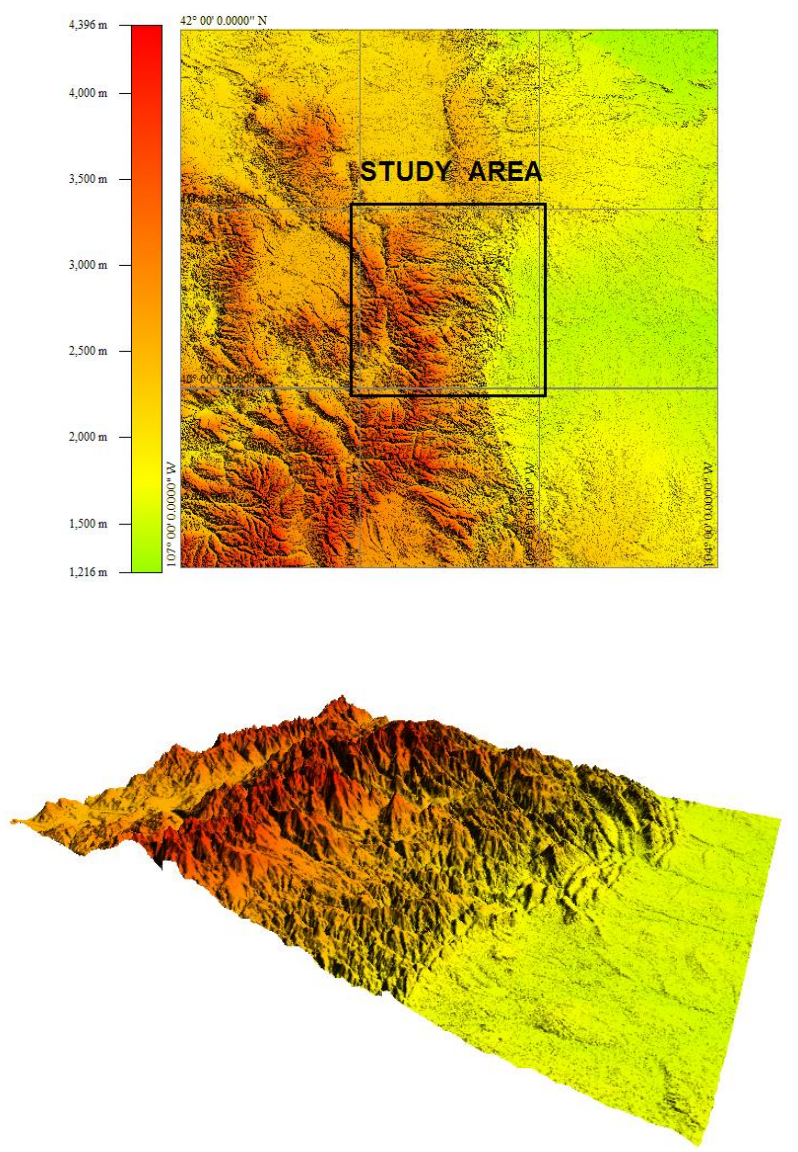

Figure (2): SRTM 30 of the Study bounded between $39^{\circ} \mathrm{N}$ to $42^{\circ} \mathrm{N}$ latitudes and $104^{\circ} \mathrm{W}$ to $107^{\circ} \mathrm{W}$ longitudes $@$ NASA [13] and USGS [17] 
The data had been loaded SRTM90 format directly included (1201 rows $* 1201$ columns), and indicated the elevations in integer values. The interpolation was carried out using the different four polynomial orders using a well-developed computer program. The results were 3601 rows $* 3601$ columns, with pixel size 0.000278 arc degree with the same area of SRTM30. This process kept the regularity of grid data for further processing.

After interpolating process, the produced SRTM30 resolution was compared with the original SRTM30 on a pixel by pixel basis concerning with the value of pixel size 0.000278 arc degrees.

Elevation differences map was computed and descriptive statistics were presented as mean elevation differences, root mean squared error, and maximum/ minimum elevation differences. Both methods of averaging and sampling had been used for the simulated data to evaluate which one was effective during the interpolation process.

The elevation differences between predicted SRTM 30 and actual SRTM30 were evaluated by the averaging method are presented in Table 1, which revealed that about $97 \%$ of the total number of pixels had elevation differences varied from zero to \pm 6 meter by using the fourth polynomial order aligned with $96 \%, 87 \%$, and $76 \%$ for the third (bicubic), second, and first polynomial orders, respectively. In addition, the elevation differences controlled by \pm 16 meter evaluated by the fourth order were about $50 \%$ less than the other types of orders.

The mean differences and RMSE were apparent different among the fourth, third, second polynomial orders. Compared with all evaluated orders, the root mean square errors of the fourth order was the smallest. It could be concluded that the fourth order polynomial had the prominent results compared with the other polynomial orders when using the averaging method.

The elevation differences between predicted SRTM 30 and actual SRTM30 were evaluated by the sampling method are presented in Table 2, which showed that about $95 \%$ of the total number of pixels had elevation differences varied from zero to \pm 6 meter by using the fourth polynomial order, aligned with $94 \%, 90 \%$, and $72 \%$ for the third (bicubic), second, and first orders, respectively. Moreover, by using the fourth order, the elevation differences controlled by \pm 16 meter were nearly $30 \%$ less than the third order while the other types of orders had a large difference.

Although the mean differences were comparable among the four orders, the RMSE for the fourth order was smaller than the other orders. It could be concluded that the fourth polynomial order had the prominent results compared with the other polynomial orders when the sampling method was used.
Notably, using the fourth polynomial order for both methods of averaging and sampling is suitable to predict or produce SRTM30 from SRTM90. In term of comparing the averaging and sampling methods, it is clear that the elevation differences varied from zero to \pm 6 meter by using averaging method of about $2 \%$ larger than sampling method. Moreover, the elevation differences controlled by \pm 16 meter were almost two times by using sampling method more than the averaging method. The mean differences of both methods were comparable while RMSE for averaging method were better than the sampling method which proves the superiority of the averaging over the sampling method.

\section{INTERPOLATION PROCESS OF REAL DATA}

The simulated data showed no great differences between the averaging and sampling method. By using the fourth polynomial order, the results of interpolation were the best. The SRTM90 data for the study area was considered with the averaging data because this data freely available while the sampling data must be ordered. Data had to be pre-processed by using SRTMFILLC[16] because there are some typical problems that could be found from the original SRTM DEM such as the existing of land data voids and large voids of the water body as shown in Figure 2.

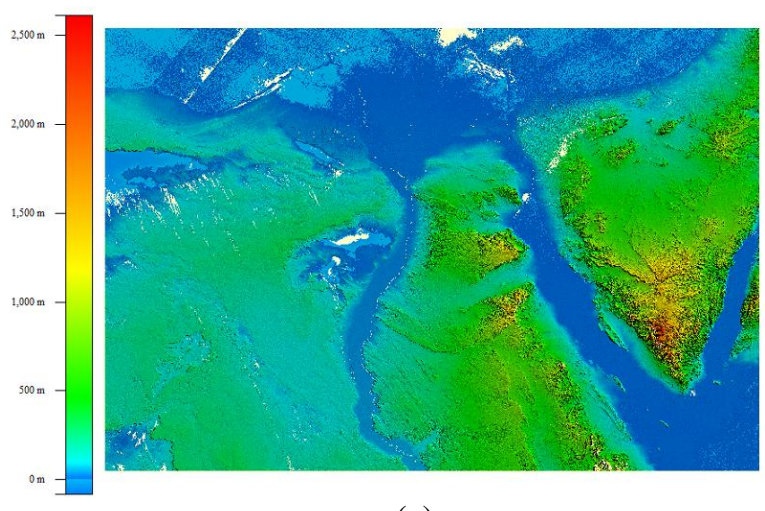

(a)

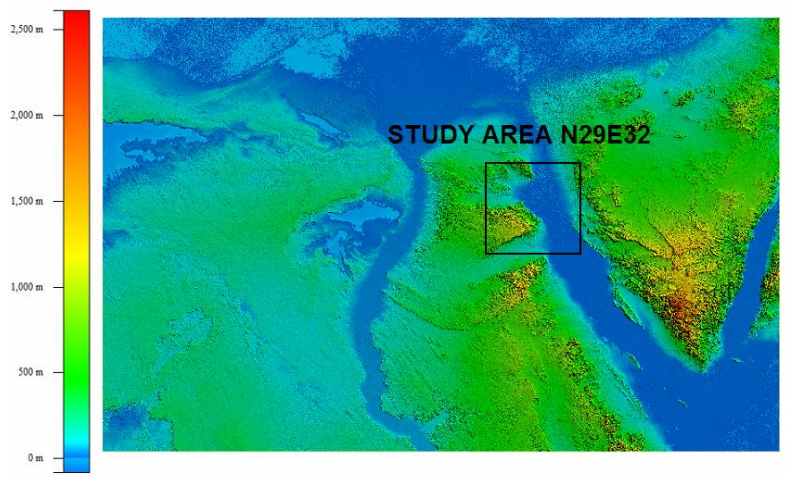

(b)

Figure (2): SRTM 90 for a part of Egypt: (a) before void filling (b) and after void filling (C) NASA [13] and USGS [17] 
Table (1): Differences between predicted SRTM 30 and real SRTM30 by using the averaging method.

\begin{tabular}{|c|c|c|c|c|c|c|c|c|}
\hline \multirow{3}{*}{$\begin{array}{c}\text { Elevation } \\
\text { Differences } \\
\text { in meter }\end{array}$} & \multicolumn{8}{|c|}{ Differences between Predicted SRTM 30 and Real SRTM30 using Averaging Method } \\
\hline & \multicolumn{2}{|c|}{ Using $1^{\text {st }}$ order } & \multicolumn{2}{|c|}{ Using $2^{\text {nd }}$ order } & \multicolumn{2}{|c|}{ Using $3^{\text {rd }}$ order } & \multicolumn{2}{|c|}{ Using $4^{\text {th }}$ order } \\
\hline & $\begin{array}{l}\text { Count } \\
\text { cells }\end{array}$ & $\%$ area & $\begin{array}{l}\text { Count } \\
\text { cells }\end{array}$ & $\%$ area & $\begin{array}{c}\text { Count } \\
\text { cells }\end{array}$ & $\%$ area & $\begin{array}{l}\text { Count } \\
\text { cells }\end{array}$ & $\%$ area \\
\hline 0.00 & 1875275 & 14.46 & 2505807 & 19.32 & 3010785 & 23.22 & 3227989 & 24.89 \\
\hline 1.00 & 2720769 & 20.98 & 3622585 & 27.94 & 4259672 & 32.85 & 4484135 & 34.58 \\
\hline 2.00 & 1767457 & 13.63 & 2202742 & 16.99 & 2369706 & 18.27 & 2355694 & 18.17 \\
\hline 3.00 & 1239041 & 9.56 & 1379674 & 10.64 & 1309607 & 10.10 & 1224745 & 9.44 \\
\hline 4.00 & 935832 & 7.22 & 911754 & 7.03 & 746035 & 5.75 & 662060 & 5.11 \\
\hline 5.00 & 739667 & 5.70 & 623402 & 4.81 & 439446 & 3.39 & 374077 & 2.88 \\
\hline 6.00 & 597129 & 4.60 & 436323 & 3.36 & 266655 & 2.06 & 219931 & 1.70 \\
\hline 7.00 & 491849 & 3.79 & 311394 & 2.40 & 167237 & 1.29 & 134806 & 1.04 \\
\hline 8.00 & 406726 & 3.14 & 225087 & 1.74 & 107888 & 0.83 & 84557 & 0.65 \\
\hline 9.00 & 339622 & 2.62 & 165403 & 1.28 & 72514 & 0.56 & 54847 & 0.42 \\
\hline 10.00 & 283174 & 2.18 & 123450 & 0.95 & 49662 & 0.38 & 36932 & 0.28 \\
\hline 11.00 & 236805 & 1.83 & 92903 & 0.72 & 34786 & 0.27 & 25364 & 0.20 \\
\hline 12.00 & 199328 & 1.54 & 70058 & 0.54 & 25478 & 0.20 & 18106 & 0.14 \\
\hline 13.00 & 167362 & 1.29 & 54414 & 0.42 & 18791 & 0.14 & 12858 & 0.10 \\
\hline 14.00 & 141109 & 1.09 & 41961 & 0.32 & 14138 & 0.11 & 9319 & 0.07 \\
\hline 15.00 & 118853 & 0.92 & 32803 & 0.25 & 11140 & 0.09 & 7079 & 0.05 \\
\hline$\geq 16$ & 707203 & 5.45 & 167441 & 1.29 & 63661 & 0.49 & 34702 & 0.27 \\
\hline Total Pixels & \multicolumn{8}{|c|}{12967201} \\
\hline \multicolumn{9}{|c|}{ Statistics } \\
\hline Mean Diff. & \multicolumn{2}{|c|}{0.0024} & \multicolumn{2}{|c|}{0.0023} & \multicolumn{2}{|c|}{0.003} & \multicolumn{2}{|c|}{0.007} \\
\hline RMSE & \multicolumn{2}{|c|}{7.599} & \multicolumn{2}{|c|}{4.700} & \multicolumn{2}{|c|}{3.420} & \multicolumn{2}{|c|}{2.943} \\
\hline Min. Elev. & \multicolumn{2}{|c|}{1472} & \multicolumn{2}{|c|}{1467} & \multicolumn{2}{|c|}{1459} & \multicolumn{2}{|c|}{1466} \\
\hline Max. Elev. & \multicolumn{2}{|c|}{4408} & \multicolumn{2}{|c|}{4486} & \multicolumn{2}{|c|}{4324} & \multicolumn{2}{|c|}{4317} \\
\hline Min. Diff. & \multicolumn{2}{|c|}{-337} & \multicolumn{2}{|c|}{-417} & \multicolumn{2}{|c|}{-321} & \multicolumn{2}{|c|}{-286} \\
\hline Max. Diff. & \multicolumn{2}{|c|}{275} & \multicolumn{2}{|c|}{278} & \multicolumn{2}{|c|}{271} & 23 & \\
\hline
\end{tabular}


Table (2): Differences between predicted SRTM 30 and real SRTM30 by using the sampling method.

\begin{tabular}{|c|c|c|c|c|c|c|c|c|}
\hline \multirow{3}{*}{$\begin{array}{c}\text { Elevation } \\
\text { Differences } \\
\text { in meter }\end{array}$} & \multicolumn{8}{|c|}{ Differences between Predicted SRTM 30 and Real SRTM30 using Sampling Method } \\
\hline & \multicolumn{2}{|c|}{ Using $1^{\text {st }}$ order } & \multicolumn{2}{|c|}{ Using $2^{\text {nd }}$ order } & \multicolumn{2}{|c|}{ Using $3^{\text {rd }}$ order } & \multicolumn{2}{|c|}{ Using $4^{\text {th }}$ order } \\
\hline & $\begin{array}{l}\text { Count } \\
\text { cells } \\
\end{array}$ & $\%$ area & $\begin{array}{c}\text { Count } \\
\text { cells }\end{array}$ & $\%$ area & $\begin{array}{c}\text { Count } \\
\text { cells }\end{array}$ & $\%$ area & $\begin{array}{l}\text { Count } \\
\text { cells }\end{array}$ & $\%$ area \\
\hline 0.00 & 2740100 & 21.13 & 3341909 & 25.77 & 3743206 & 28.87 & 3910792 & 30.16 \\
\hline 1.00 & 2147455 & 16.56 & 3005505 & 23.18 & 3516013 & 27.11 & 3691637 & 28.47 \\
\hline 2.00 & 1609800 & 12.41 & 2020558 & 15.58 & 2161454 & 16.67 & 2151650 & 16.59 \\
\hline 3.00 & 1197240 & 9.23 & 1320419 & 10.18 & 1269847 & 9.79 & 1208293 & 9.32 \\
\hline 4.00 & 920311 & 7.10 & 887314 & 6.84 & 760733 & 5.87 & 695325 & 5.36 \\
\hline 5.00 & 732946 & 5.65 & 611485 & 4.72 & 469569 & 3.62 & 419076 & 3.23 \\
\hline 6.00 & 592230 & 4.57 & 431214 & 3.33 & 299134 & 2.31 & 262919 & 2.03 \\
\hline 7.00 & 485494 & 3.74 & 310363 & 2.39 & 196619 & 1.52 & 171695 & 1.32 \\
\hline 8.00 & 400431 & 3.09 & 226427 & 1.75 & 133439 & 1.03 & 116516 & 0.90 \\
\hline 9.00 & 333516 & 2.57 & 168830 & 1.30 & 93027 & 0.72 & 80592 & 0.62 \\
\hline 10.00 & 276786 & 2.13 & 127296 & 0.98 & 66643 & 0.51 & 57657 & 0.44 \\
\hline 11.00 & 231016 & 1.78 & 96733 & 0.75 & 49042 & 0.38 & 42144 & 0.33 \\
\hline 12.00 & 193740 & 1.49 & 75295 & 0.58 & 36833 & 0.28 & 31386 & 0.24 \\
\hline 13.00 & 162836 & 1.26 & 58493 & 0.45 & 28095 & 0.22 & 23632 & 0.18 \\
\hline 14.00 & 137331 & 1.06 & 45868 & 0.35 & 22054 & 0.17 & 18263 & 0.14 \\
\hline 15.00 & 114980 & 0.89 & 36696 & 0.28 & 17479 & 0.13 & 14053 & 0.11 \\
\hline$\geq 16$ & 690989 & 5.33 & 202796 & 1.56 & 104014 & 0.80 & 71571 & 0.55 \\
\hline Total Pixels & \multicolumn{8}{|c|}{12967201} \\
\hline \multicolumn{9}{|c|}{ Statistics } \\
\hline Mean Diff. & \multicolumn{2}{|c|}{-0.001} & \multicolumn{2}{|c|}{-0.001} & \multicolumn{2}{|c|}{-0.003} & \multicolumn{2}{|c|}{0.004} \\
\hline RMSE & \multicolumn{2}{|c|}{7.683} & \multicolumn{2}{|c|}{5.103} & \multicolumn{2}{|c|}{4.117} & \multicolumn{2}{|c|}{3.666} \\
\hline Min. Elev. & \multicolumn{2}{|c|}{1472} & \multicolumn{2}{|c|}{1468} & \multicolumn{2}{|c|}{1459} & \multicolumn{2}{|c|}{1456} \\
\hline Max. Elev. & \multicolumn{2}{|c|}{4422} & \multicolumn{2}{|c|}{4592} & \multicolumn{2}{|c|}{4344} & \multicolumn{2}{|c|}{4706} \\
\hline Min. Diff. & \multicolumn{2}{|c|}{-329} & \multicolumn{2}{|c|}{-523} & \multicolumn{2}{|c|}{-446} & \multicolumn{2}{|c|}{-835} \\
\hline Max. Diff. & \multicolumn{2}{|c|}{277} & \multicolumn{2}{|c|}{297} & \multicolumn{2}{|c|}{359} & 45 & \\
\hline
\end{tabular}




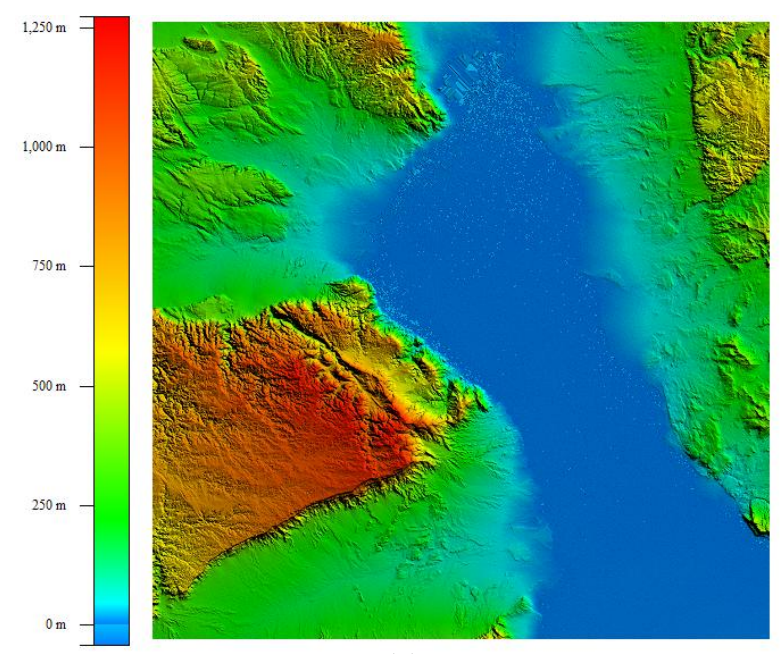

(a)

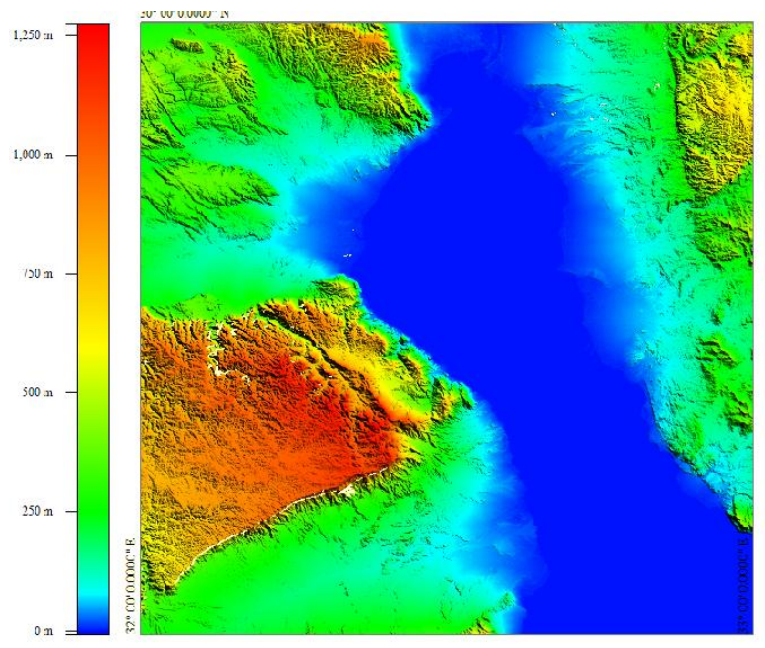

(b)

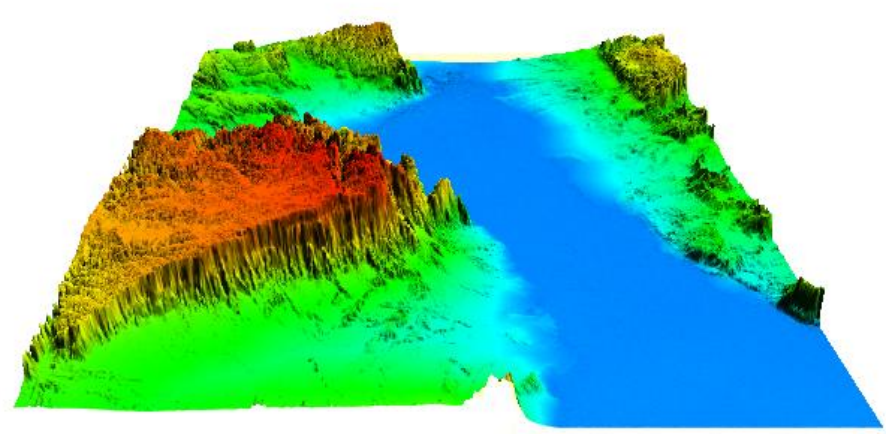

Figure (3): SRTM90 of Study Area N29E32 (C) NASA a) SRTM90 version_1, b) SRTM90 version_2

In addition the presence of some voids over land surfaces, there are large voids of the water body for oceans, lakes, and seas, especially the coastlines which can be noticed in Figure 3.a. The coastlines of oceans, and seas had been edited and improved and became available in SRTM30 version 2 [18], [19] and [20]. The study area of SRTM30 version 2 presented in Figure 3.b.
SRTM90 of real data was concerned with a region of EGYPT extended from $26^{\circ} \mathrm{N}$ to $27^{\circ} \mathrm{N}$ latitude and $32^{\circ} \mathrm{E}$ to $33^{\circ} \mathrm{E}$ longitude. This area was presented by N29E32 of SRTM90 DEM within EGYPT that can be freely downloaded and covered one degree by one degree squared to have an area of $10776 \mathrm{~km}^{2}$ (1201 rows*1201columns) with pixel size 0.000833 arc degree. The elevations varied from a minimum of -43 meter to a maximum of 1247 meter as presented in Figure 3. Data had to be pre-processed by using SRTMFILL(C) [16] to fill the existing of data voids that could be found from the original SRTM90 DEM as shown in Figures 2 and $3 \mathrm{~b}$.

The results of SRTM30 using different four orders polynomial interpolation of SRTM90-version_1 are shown in Figure 4. The elevation differences between predicted SRTM30 and real SRTM90 together with their relevant statistics are presented in Table 3 and Figure 5. It can be noticed that more than $97 \%$ of the total number of pixels had elevation differences varied from zero to \pm 6 meter by using the fourth polynomial order, aligned with about $97 \%, 96 \%$, and $92 \%$ for the third, second, and first orders, respectively. In addition, by using fourth polynomial order, the elevation differences controlled by \pm 16 meter were less than the third, second, and first polynomial orders. The differences of means, and RMSE achieved by the fourth order, aligned with third, second, and first orders were obvious.

It could be concluded that the interpolation resulted in the best results compared with the other polynomial orders when the fourth order polynomial was used.

The elevation differences between predicted SRTM30 and real editing SRTM90 version_2 together with their relevant statistics are presented in Table 4. It is clearly that further than $11 \%$ of the total number of pixels had elevation differences zero value by using the editing SRTM90 version_2 further non editing version_1 based on the presence of red sea. In addition it is clear that the results had slightly improved by using editing version. It was clear that the fourth order polynomial had the better results than other polynomial degrees. 


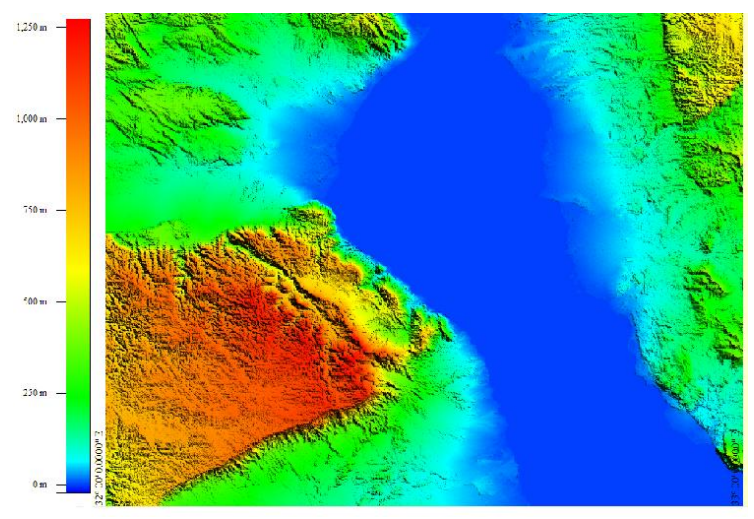

(a)

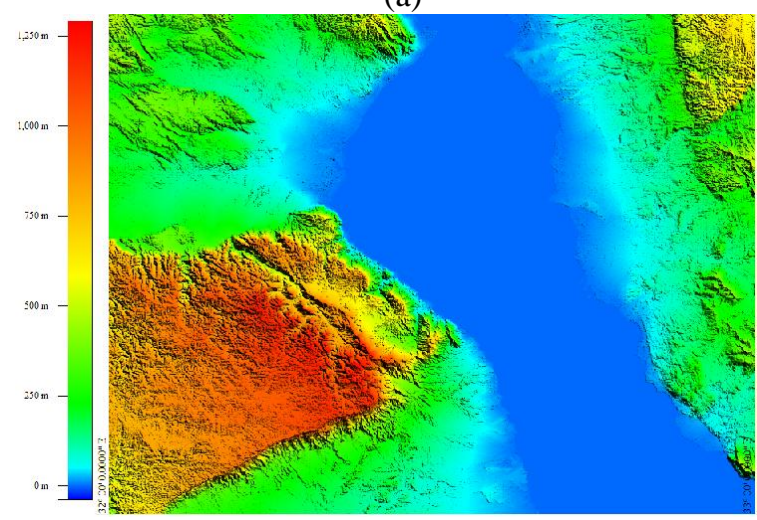

(b)

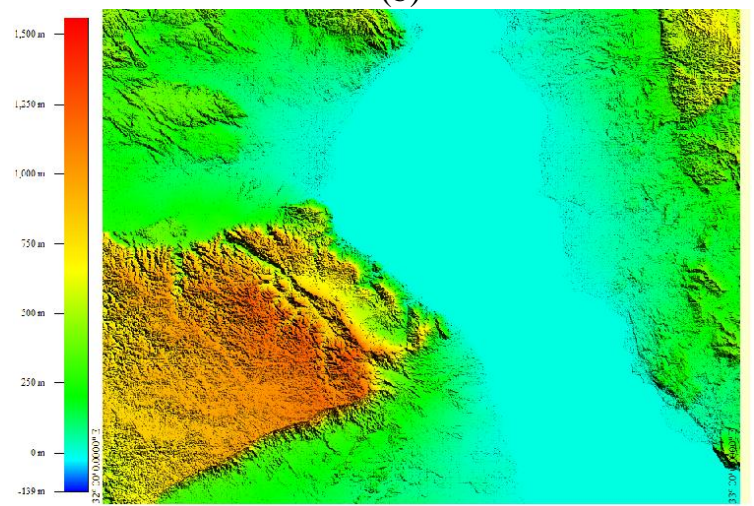

(c)

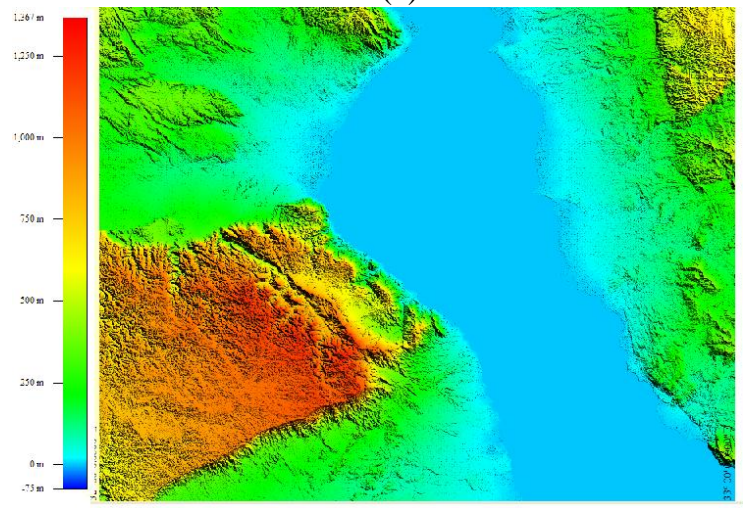

(d)

Figure (4): The results of created SRTM30 for the study area a) by using $1^{\text {st }}$ order polynomial b) by using $2^{\text {nd }}$ polynomial order c) by using $3^{\text {rd }}$ polynomial order d) by using $4^{\text {th }}$ polynomial order.

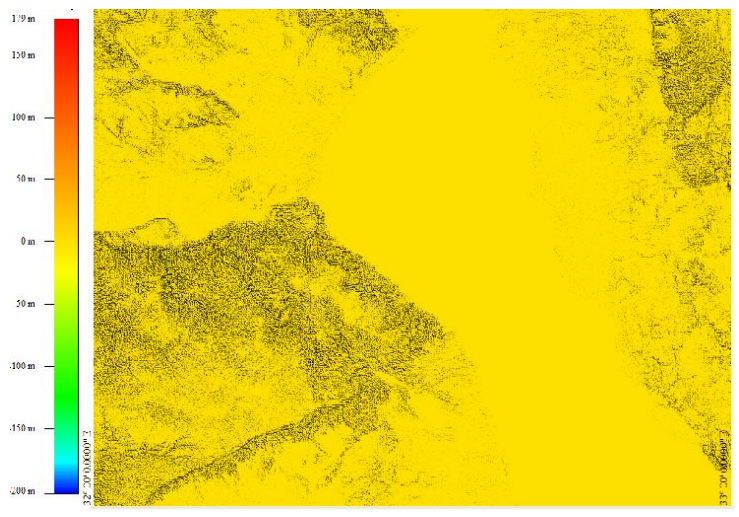

(a)

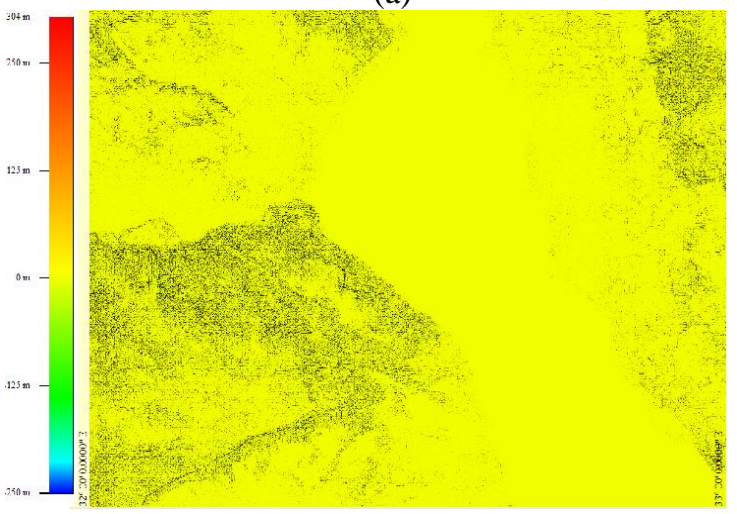

(b)

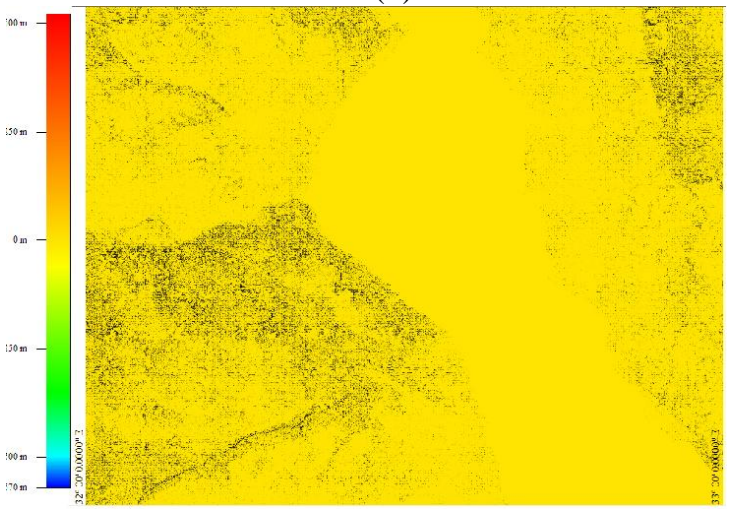

(c)

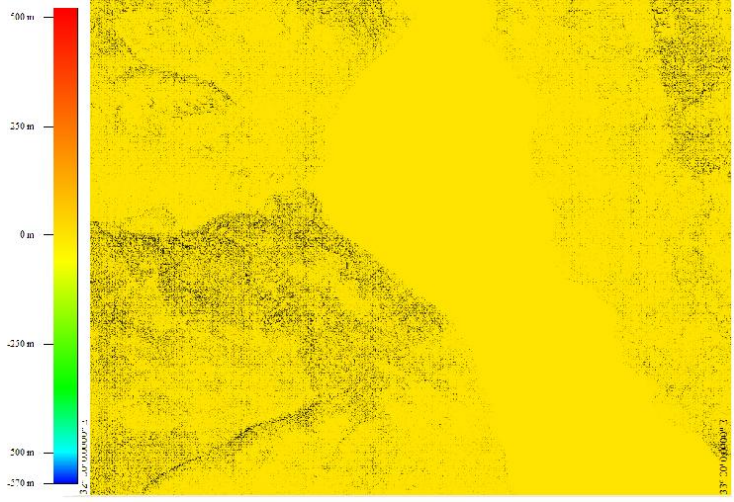

(d)

Figure (5): Elevation differences between created SRTM30 and SRTM90 for the study area a) by using $1^{\text {st }}$ order polynomial $b$ ) by using $2^{\text {nd }}$ polynomial order c) by using $3^{\text {rd }}$ polynomial order d) by using $4^{\text {th }}$ polynomial order. 
Table (3): The elevations differences between predicted SRTM 30 using four orders of Polynomial interpolation and original SRTM 90 version_1

\begin{tabular}{|c|c|c|c|c|c|c|c|c|}
\hline \multirow{3}{*}{$\begin{array}{c}\text { Elevation } \\
\text { Differences } \\
\text { in meter }\end{array}$} & \multicolumn{8}{|c|}{ Differences between Predicted SRTM 30 and SRTM 90} \\
\hline & \multicolumn{2}{|c|}{ Using $1^{\text {st }}$ order } & \multicolumn{2}{|c|}{ Using $2^{\text {nd }}$ order } & \multicolumn{2}{|c|}{ Using $3^{\text {rd }}$ order } & \multicolumn{2}{|c|}{ Using $4^{\text {th }}$ order } \\
\hline & $\begin{array}{l}\text { Count } \\
\text { cells } \\
\end{array}$ & $\%$ area & $\begin{array}{c}\text { Count } \\
\text { cells }\end{array}$ & $\%$ area & $\begin{array}{c}\text { Count } \\
\text { cells }\end{array}$ & $\%$ area & $\begin{array}{l}\text { Count } \\
\text { cells }\end{array}$ & $\%$ area \\
\hline 0.00 & 4986798 & 38.46 & 5909978 & 45.58 & 6274674 & 48.39 & 6396836 & 49.33 \\
\hline 1.00 & 3982313 & 30.71 & 3979952 & 30.69 & 3882342 & 29.94 & 3863691 & 29.80 \\
\hline 2.00 & 1362593 & 10.51 & 1173277 & 9.05 & 1180494 & 9.10 & 1191180 & 9.19 \\
\hline 3.00 & 662089 & 5.11 & 574068 & 4.43 & 569522 & 4.39 & 556986 & 4.30 \\
\hline 4.00 & 424294 & 3.27 & 352777 & 2.72 & 326535 & 2.52 & 307710 & 2.37 \\
\hline 5.00 & 304407 & 2.35 & 236400 & 1.82 & 203873 & 1.57 & 187813 & 1.45 \\
\hline 6.00 & 230964 & 1.78 & 166871 & 1.29 & 133631 & 1.03 & 121569 & 0.94 \\
\hline 7.00 & 179737 & 1.39 & 121648 & 0.94 & 91865 & 0.71 & 82610 & 0.64 \\
\hline 8.00 & 142445 & 1.10 & 90580 & 0.70 & 65193 & 0.50 & 58501 & 0.45 \\
\hline 9.00 & 115301 & 0.89 & 68005 & 0.52 & 46909 & 0.36 & 42401 & 0.33 \\
\hline 10.00 & 93579 & 0.72 & 52595 & 0.41 & 34814 & 0.27 & 31662 & 0.24 \\
\hline 11.00 & 76042 & 0.59 & 40807 & 0.31 & 26241 & 0.20 & 23999 & 0.19 \\
\hline 12.00 & 62408 & 0.48 & 32473 & 0.25 & 20452 & 0.16 & 18492 & 0.14 \\
\hline 13.00 & 52026 & 0.40 & 25508 & 0.20 & 15939 & 0.12 & 14240 & 0.11 \\
\hline 14.00 & 43203 & 0.33 & 20344 & 0.16 & 12952 & 0.10 & 11286 & 0.09 \\
\hline 15.00 & 35778 & 0.28 & 16623 & 0.13 & 10392 & 0.08 & 9140 & 0.07 \\
\hline$\geq 16$ & 213224 & 1.64 & 105295 & 0.81 & 71373 & 0.55 & 49085 & 0.38 \\
\hline Total Pixels & \multicolumn{8}{|c|}{12967201} \\
\hline \multicolumn{9}{|c|}{ Statistics } \\
\hline Mean Diff. & \multicolumn{2}{|c|}{-0.0030} & \multicolumn{2}{|c|}{0.0109} & \multicolumn{2}{|c|}{0.0016} & \multicolumn{2}{|c|}{0.0012} \\
\hline RMSE & \multicolumn{2}{|c|}{4.567} & \multicolumn{2}{|c|}{3.595} & \multicolumn{2}{|c|}{3.213} & \multicolumn{2}{|c|}{2.756} \\
\hline Min. Elev. & \multicolumn{2}{|c|}{-43} & \multicolumn{2}{|c|}{-54} & \multicolumn{2}{|c|}{-138} & \multicolumn{2}{|c|}{-77} \\
\hline Max. Elev. & \multicolumn{2}{|c|}{1274} & \multicolumn{2}{|c|}{1292} & \multicolumn{2}{|c|}{1559} & \multicolumn{2}{|c|}{1365} \\
\hline Min. Diff. & \multicolumn{2}{|c|}{-179} & \multicolumn{2}{|c|}{-248} & \multicolumn{2}{|c|}{-521} & \multicolumn{2}{|c|}{-521} \\
\hline Max. Diff. & \multicolumn{2}{|c|}{203} & \multicolumn{2}{|c|}{315} & \multicolumn{2}{|c|}{569} & 32 & \\
\hline
\end{tabular}


Table (4): The elevations differences between predicted SRTM 30 using four orders of Polynomial interpolation and original editing SRTM 90 version_2

\begin{tabular}{|c|c|c|c|c|c|c|c|c|}
\hline \multirow{3}{*}{$\begin{array}{c}\text { Elevation } \\
\text { Differences } \\
\text { in meter }\end{array}$} & \multicolumn{8}{|c|}{ Differences between Predicted SRTM 30 and SRTM 90} \\
\hline & \multicolumn{2}{|c|}{ Using $1^{\text {st }}$ order } & \multicolumn{2}{|c|}{ Using $2^{\text {nd }}$ order } & \multicolumn{2}{|c|}{ Using $3^{\text {rd }}$ order } & \multicolumn{2}{|c|}{ Using $4^{\text {th }}$ order } \\
\hline & $\begin{array}{l}\text { Count } \\
\text { cells }\end{array}$ & $\%$ area & $\begin{array}{c}\text { Count } \\
\text { cells }\end{array}$ & $\%$ area & $\begin{array}{c}\text { Count } \\
\text { cells }\end{array}$ & $\%$ area & $\begin{array}{c}\text { Count } \\
\text { cells }\end{array}$ & $\%$ area \\
\hline 0.00 & 6684350 & 51.55 & 7413485 & 57.17 & 7722787 & 59.56 & 7856031 & 60.58 \\
\hline 1.00 & 2672692 & 20.61 & 2801584 & 21.61 & 2813945 & 21.70 & 2810300 & 21.67 \\
\hline 2.00 & 1078711 & 8.32 & 961051 & 7.41 & 963183 & 7.43 & 952217 & 7.34 \\
\hline 3.00 & 597960 & 4.61 & 513364 & 3.96 & 492275 & 3.80 & 472354 & 3.64 \\
\hline 4.00 & 403266 & 3.11 & 328289 & 2.53 & 291679 & 2.25 & 271053 & 2.09 \\
\hline 5.00 & 295511 & 2.28 & 225108 & 1.74 & 185652 & 1.43 & 169555 & 1.31 \\
\hline 6.00 & 226467 & 1.75 & 161010 & 1.24 & 124039 & 0.96 & 111791 & 0.86 \\
\hline 7.00 & 177343 & 1.37 & 118128 & 0.91 & 85438 & 0.66 & 77215 & 0.60 \\
\hline 8.00 & 141583 & 1.09 & 88298 & 0.68 & 60781 & 0.47 & 54940 & 0.42 \\
\hline 9.00 & 114687 & 0.88 & 66816 & 0.52 & 44160 & 0.34 & 39822 & 0.31 \\
\hline 10.00 & 93070 & 0.72 & 51813 & 0.40 & 32761 & 0.25 & 29965 & 0.23 \\
\hline 11.00 & 75991 & 0.59 & 40221 & 0.31 & 24727 & 0.19 & 23057 & 0.18 \\
\hline 12.00 & 62126 & 0.48 & 32048 & 0.25 & 19366 & 0.15 & 17770 & 0.14 \\
\hline 13.00 & 51966 & 0.40 & 25088 & 0.19 & 15235 & 0.12 & 13584 & 0.10 \\
\hline 14.00 & 43013 & 0.33 & 20195 & 0.16 & 12216 & 0.09 & 10889 & 0.08 \\
\hline 15.00 & 35616 & 0.27 & 16413 & 0.13 & 9892 & 0.08 & 8849 & 0.07 \\
\hline$\geq 16$ & 212849 & 1.64 & 104290 & 0.80 & 69065 & 0.53 & 47809 & 0.37 \\
\hline Total Pixels & \multicolumn{8}{|c|}{12967201} \\
\hline \multicolumn{9}{|c|}{ Statistics } \\
\hline Mean Diff. & \multicolumn{2}{|c|}{0.008} & \multicolumn{2}{|c|}{0.010} & \multicolumn{2}{|c|}{0.002} & \multicolumn{2}{|c|}{-0.001} \\
\hline RMSE & \multicolumn{2}{|c|}{4.526} & \multicolumn{2}{|c|}{3.538} & \multicolumn{2}{|c|}{3.122} & \multicolumn{2}{|c|}{2.66} \\
\hline Min. Elev. & \multicolumn{2}{|c|}{-43} & \multicolumn{2}{|c|}{-54} & \multicolumn{2}{|c|}{-138} & \multicolumn{2}{|c|}{-77} \\
\hline Max. Elev. & \multicolumn{2}{|c|}{1274} & \multicolumn{2}{|c|}{1292} & \multicolumn{2}{|c|}{1559} & \multicolumn{2}{|c|}{1365} \\
\hline Min. Diff. & \multicolumn{2}{|c|}{-179} & \multicolumn{2}{|c|}{-248} & \multicolumn{2}{|c|}{-521} & \multicolumn{2}{|c|}{-521} \\
\hline Max. Diff. & \multicolumn{2}{|c|}{203} & \multicolumn{2}{|c|}{315} & \multicolumn{2}{|c|}{569} & & \\
\hline
\end{tabular}




\section{GROUND STUDY AREA}

In order to evaluate the accuracy of the converting SRTM 90 to SRTM 30, comparisons with data SRTM 90 and interpolated data were performed concerned with the ground truth area which has almost 13 $\mathrm{km}^{2}$ area and presented in figure 6 . This area varied in topography, ranged from mean sea level to height $40 \mathrm{~m}$. SRTM90 and predicted SRTM 30 can be evaluated depending on the structure of land topography area from field measurements. Although the scale of the studied area is quite small; about $13 \mathrm{~km}^{2}$, it was used for testing the SRTM90 and predicted SRTM30 data.

This area has irregular shape that represented a significant difficulty in exporting data process so that more concern in the converting process was required. As a result, the indicators showed that the success of predicted SRTM are not uniform, the statistics did not show a significant difference between the original SRTM 90 and the tested data. Also, the results showed that SRTM90 had better performance than the predicted SRTM30 (Figures 7 and Table 5, and 6). Also these results obtained if the real data which consists of 1100 observations used instead of terrain surface (Figure 8).
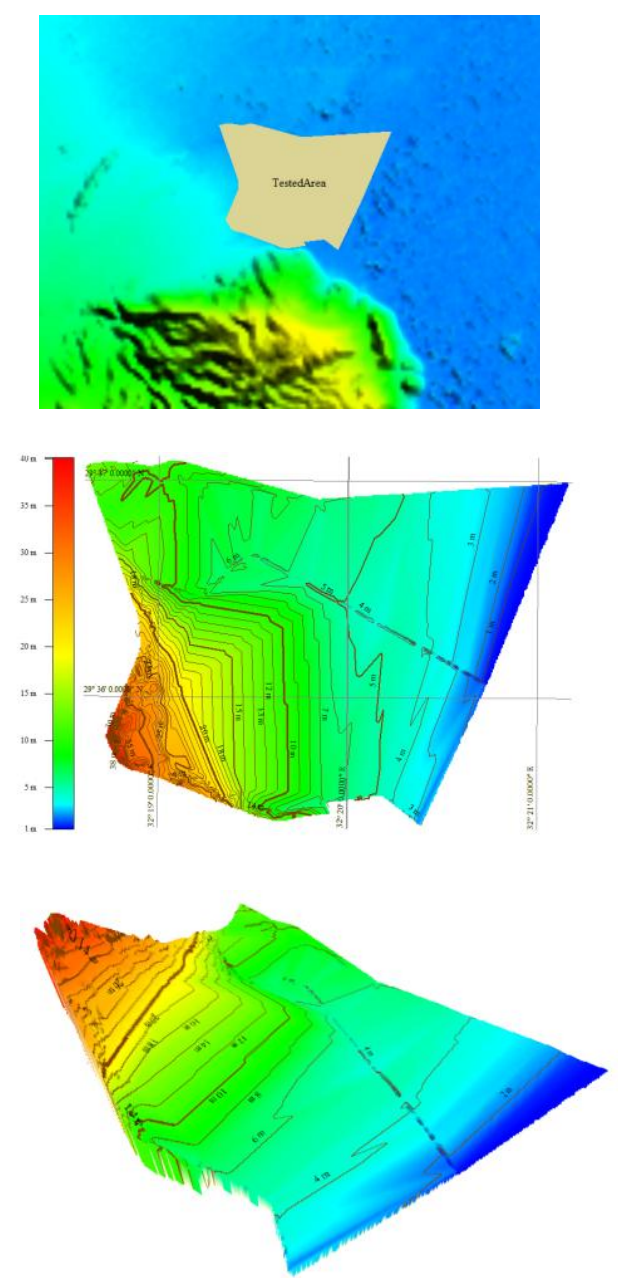

Figure (6): The study area
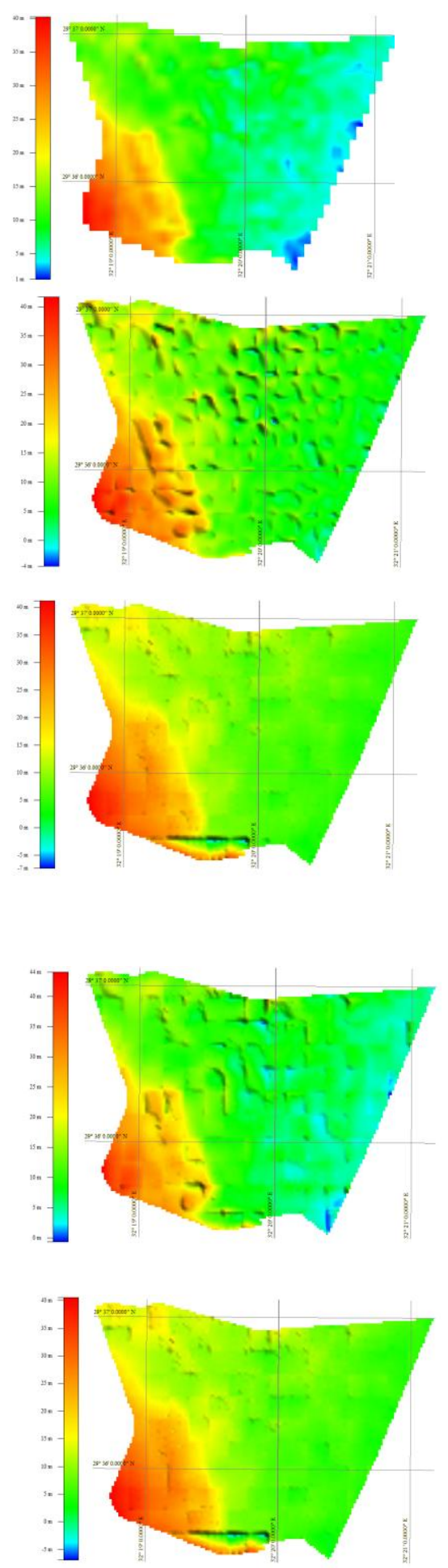

Figure (7): from above to bottom the results of elevation differences for study area between real data and SRTM90, and between real data and predicted SRTM30 by using ( 4 th, $3^{\text {rd }}, 2^{\text {nd }}$, and $1^{\text {st }}$ polynomial order). 
Table (5): Study area elevations difference between predicted SRTM 30 using different orders of polynomial interpolation, original non editing SRTM90 (version_1), and real data

\begin{tabular}{|c|c|c|c|c|c|c|c|c|c|c|}
\hline \multirow{3}{*}{$\begin{array}{l}\text { Elevation } \\
\text { Differences } \\
\text { in meter }\end{array}$} & \multicolumn{10}{|c|}{ Differences between Predicted Tested Area and ( Predicted SRTM 30, SRTM 90) } \\
\hline & \multicolumn{2}{|c|}{ SRTM 90} & \multicolumn{2}{|c|}{ Using $1^{\text {st }}$ order } & \multicolumn{2}{|c|}{ Using $2^{\text {nd }}$ order } & \multicolumn{2}{|c|}{ Using $3^{\text {rd }}$ order } & \multicolumn{2}{|c|}{ Using $4^{\text {th }}$ order } \\
\hline & $\begin{array}{l}\text { Count } \\
\text { cells }\end{array}$ & $\%$ area & $\begin{array}{l}\text { Count } \\
\text { cells }\end{array}$ & $\%$ area & $\begin{array}{l}\text { Count } \\
\text { cells }\end{array}$ & $\%$ area & $\begin{array}{l}\text { Count } \\
\text { cells }\end{array}$ & $\%$ area & $\begin{array}{l}\text { Count } \\
\text { cells }\end{array}$ & $\%$ area \\
\hline $0-1$ & 173725 & 38.62 & 170490 & 37.90 & 175114 & 38.93 & 173044 & 38.47 & 175041 & 38.91 \\
\hline $1 \sim 2$ & 59321 & 13.19 & 56336 & 12.52 & 57304 & 12.74 & 60311 & 13.41 & 59858 & 13.31 \\
\hline $2 \sim 3$ & 61538 & 13.68 & 60269 & 13.40 & 60695 & 13.49 & 60091 & 13.36 & 60078 & 13.35 \\
\hline $3 \sim 4$ & 56331 & 12.52 & 61511 & 13.67 & 54902 & 12.20 & 53443 & 11.88 & 51532 & 11.45 \\
\hline $4 \sim 5$ & 40801 & 9.07 & 43690 & 9.71 & 39951 & 8.88 & 38288 & 8.51 & 36920 & 8.21 \\
\hline $5 \sim 6$ & 23471 & 5.22 & 26436 & 5.88 & 24165 & 5.37 & 24204 & 5.38 & 24209 & 5.38 \\
\hline $6 \sim 7$ & 14327 & 3.18 & 13117 & 2.92 & 16138 & 3.59 & 14560 & 3.24 & 15989 & 3.55 \\
\hline $7 \sim 8$ & 8259 & 1.84 & 7929 & 1.76 & 8510 & 1.89 & 9659 & 2.15 & 9914 & 2.20 \\
\hline $8 \sim 9$ & 5352 & 1.19 & 4568 & 1.02 & 5348 & 1.19 & 6307 & 1.40 & 5799 & 1.29 \\
\hline $9 \sim 10$ & 3142 & 0.70 & 2334 & 0.52 & 3272 & 0.73 & 3716 & 0.83 & 3933 & 0.87 \\
\hline $10 \sim 11$ & 1765 & 0.39 & 784 & 0.17 & 2290 & 0.51 & 2353 & 0.52 & 2630 & 0.58 \\
\hline $11 \sim 12$ & 1092 & 0.24 & 409 & 0.09 & 1037 & 0.23 & 1616 & 0.36 & 1669 & 0.37 \\
\hline $12 \sim 13$ & 502 & 0.11 & 338 & 0.08 & 541 & 0.12 & 957 & 0.21 & 1050 & 0.23 \\
\hline $13 \sim 14$ & 176 & 0.04 & 277 & 0.06 & 310 & 0.07 & 650 & 0.14 & 727 & 0.16 \\
\hline $14 \sim 15$ & 52 & 0.01 & 345 & 0.08 & 155 & 0.03 & 332 & 0.07 & 356 & 0.08 \\
\hline $15 \sim 16$ & 5 & 0.00 & 286 & 0.06 & 66 & 0.01 & 120 & 0.03 & 82 & 0.02 \\
\hline$>16$ & 6 & 0.00 & 746 & 0.17 & 67 & 0.01 & 214 & 0.05 & 78 & 0.02 \\
\hline Total Pixels & \multicolumn{10}{|c|}{449865} \\
\hline \multicolumn{11}{|c|}{ Statistics } \\
\hline Mean Diff. & \multicolumn{2}{|c|}{-1.872} & \multicolumn{2}{|c|}{-1.877} & \multicolumn{2}{|c|}{-1.878} & \multicolumn{2}{|c|}{-1.865} & \multicolumn{2}{|c|}{-1.853} \\
\hline RMSE & \multicolumn{2}{|c|}{2.979} & \multicolumn{2}{|c|}{3.042} & \multicolumn{2}{|c|}{3.053} & \multicolumn{2}{|c|}{3.176} & \multicolumn{2}{|c|}{3.184} \\
\hline Min. Elev. & \multicolumn{2}{|c|}{-1.23} & \multicolumn{2}{|c|}{-6.90} & \multicolumn{2}{|c|}{-0.65} & \multicolumn{2}{|c|}{-7.34} & \multicolumn{2}{|c|}{-4.40} \\
\hline Max. Elev. & \multicolumn{2}{|c|}{40.21} & \multicolumn{2}{|c|}{41} & \multicolumn{2}{|c|}{44} & \multicolumn{2}{|c|}{41.18} & \multicolumn{2}{|c|}{41.70} \\
\hline Min. Diff. & \multicolumn{2}{|c|}{-16.0} & & & -16 & & -2 & & -1 & \\
\hline Max. Diff. & & 0 & & .0 & 5 & & 15 & & 8.7 & \\
\hline
\end{tabular}


Table (6): Study area elevations difference of the between predicted SRTM 30 using different orders of polynomial interpolation, original editing SRTM90 (version_2), and real data

\begin{tabular}{|c|c|c|c|c|c|c|c|c|c|c|}
\hline \multirow{3}{*}{$\begin{array}{l}\text { Elevation } \\
\text { Differences } \\
\text { in meter }\end{array}$} & \multicolumn{10}{|c|}{ Differences between Predicted Tested Area and ( Predicted SRTM 30, SRTM 90) } \\
\hline & \multicolumn{2}{|c|}{ SRTM 90} & \multicolumn{2}{|c|}{ Using $1^{\text {st }}$ order } & \multicolumn{2}{|c|}{ Using $2^{\text {nd }}$ order } & \multicolumn{2}{|c|}{ Using $3^{\text {rd }}$ order } & \multicolumn{2}{|c|}{ Using $4^{\text {th }}$ order } \\
\hline & $\begin{array}{l}\text { Count } \\
\text { cells }\end{array}$ & $\%$ area & $\begin{array}{l}\text { Count } \\
\text { cells }\end{array}$ & $\%$ area & $\begin{array}{l}\text { Count } \\
\text { cells }\end{array}$ & $\%$ area & $\begin{array}{l}\text { Count } \\
\text { cells }\end{array}$ & $\%$ area & $\begin{array}{l}\text { Count } \\
\text { cells }\end{array}$ & $\%$ area \\
\hline $0-1$ & 179464 & 39.89 & 174262 & 38.74 & 174584 & 38.81 & 170623 & 37.93 & 176402 & 39.21 \\
\hline $1 \sim 2$ & 53572 & 11.91 & 54779 & 12.18 & 58458 & 12.99 & 54472 & 12.11 & 59424 & 13.21 \\
\hline $2 \sim 3$ & 60553 & 13.46 & 57340 & 12.75 & 60372 & 13.42 & 59390 & 13.20 & 60979 & 13.55 \\
\hline $3 \sim 4$ & 60793 & 13.51 & 63864 & 14.20 & 55676 & 12.38 & 63360 & 14.08 & 52802 & 11.74 \\
\hline $4 \sim 5$ & 41623 & 9.25 & 44901 & 9.98 & 39554 & 8.79 & 45974 & 10.22 & 36833 & 8.19 \\
\hline $5 \sim 6$ & 23887 & 5.31 & 23761 & 5.28 & 25057 & 5.57 & 24657 & 5.48 & 23921 & 5.32 \\
\hline $6 \sim 7$ & 13502 & 3.00 & 12431 & 2.76 & 15045 & 3.34 & 12644 & 2.81 & 14947 & 3.32 \\
\hline $7 \sim 8$ & 6996 & 1.56 & 7272 & 1.62 & 8894 & 1.98 & 7307 & 1.62 & 9346 & 2.08 \\
\hline $8 \sim 9$ & 5388 & 1.20 & 5379 & 1.20 & 4940 & 1.10 & 5464 & 1.21 & 5306 & 1.18 \\
\hline $9 \sim 10$ & 2480 & 0.55 & 2541 & 0.56 & 3329 & 0.74 & 2156 & 0.48 & 3757 & 0.84 \\
\hline $10 \sim 11$ & 1082 & 0.24 & 1304 & 0.29 & 2136 & 0.47 & 436 & 0.10 & 2529 & 0.56 \\
\hline $11 \sim 12$ & 340 & 0.08 & 305 & 0.07 & 1021 & 0.23 & 297 & 0.07 & 1733 & 0.39 \\
\hline $12 \sim 13$ & 185 & 0.04 & 309 & 0.07 & 467 & 0.10 & 308 & 0.07 & 887 & 0.20 \\
\hline $13 \sim 14$ & 0 & 0.00 & 352 & 0.08 & 215 & 0.05 & 358 & 0.08 & 629 & 0.14 \\
\hline $14 \sim 15$ & 0 & 0.00 & 378 & 0.08 & 71 & 0.02 & 370 & 0.08 & 324 & 0.07 \\
\hline $15 \sim 16$ & 0 & 0.00 & 213 & 0.05 & 41 & 0.01 & 217 & 0.05 & 23 & 0.01 \\
\hline$>16$ & 0 & 0.00 & 474 & 0.11 & 5 & 0.00 & 479 & 0.11 & 23 & 0.01 \\
\hline Total Pixels & \multicolumn{10}{|c|}{449865} \\
\hline \multicolumn{11}{|c|}{ Statistics } \\
\hline Mean Diff. & \multicolumn{2}{|c|}{-1.849} & \multicolumn{2}{|c|}{-1.867} & \multicolumn{2}{|c|}{-1.875} & \multicolumn{2}{|c|}{-1.866} & \multicolumn{2}{|c|}{-1.849} \\
\hline RMSE & \multicolumn{2}{|c|}{2.851} & \multicolumn{2}{|c|}{3.009} & \multicolumn{2}{|c|}{3.015} & \multicolumn{2}{|c|}{2.987} & \multicolumn{2}{|c|}{3.119} \\
\hline Min. Elev. & \multicolumn{2}{|c|}{1.00} & \multicolumn{2}{|c|}{-7.00} & \multicolumn{2}{|c|}{-1.00} & \multicolumn{2}{|c|}{-3.00} & \multicolumn{2}{|c|}{-4.00} \\
\hline Max. Elev. & \multicolumn{2}{|c|}{41.00} & \multicolumn{2}{|c|}{41.00} & \multicolumn{2}{|c|}{41.00} & \multicolumn{2}{|c|}{43.00} & \multicolumn{2}{|c|}{42.00} \\
\hline Min. Diff. & \multicolumn{2}{|c|}{-12.80} & & & -19 & & -23 & & -20 & \\
\hline Max. Diff. & & & & & 6. & & 11. & & & \\
\hline
\end{tabular}




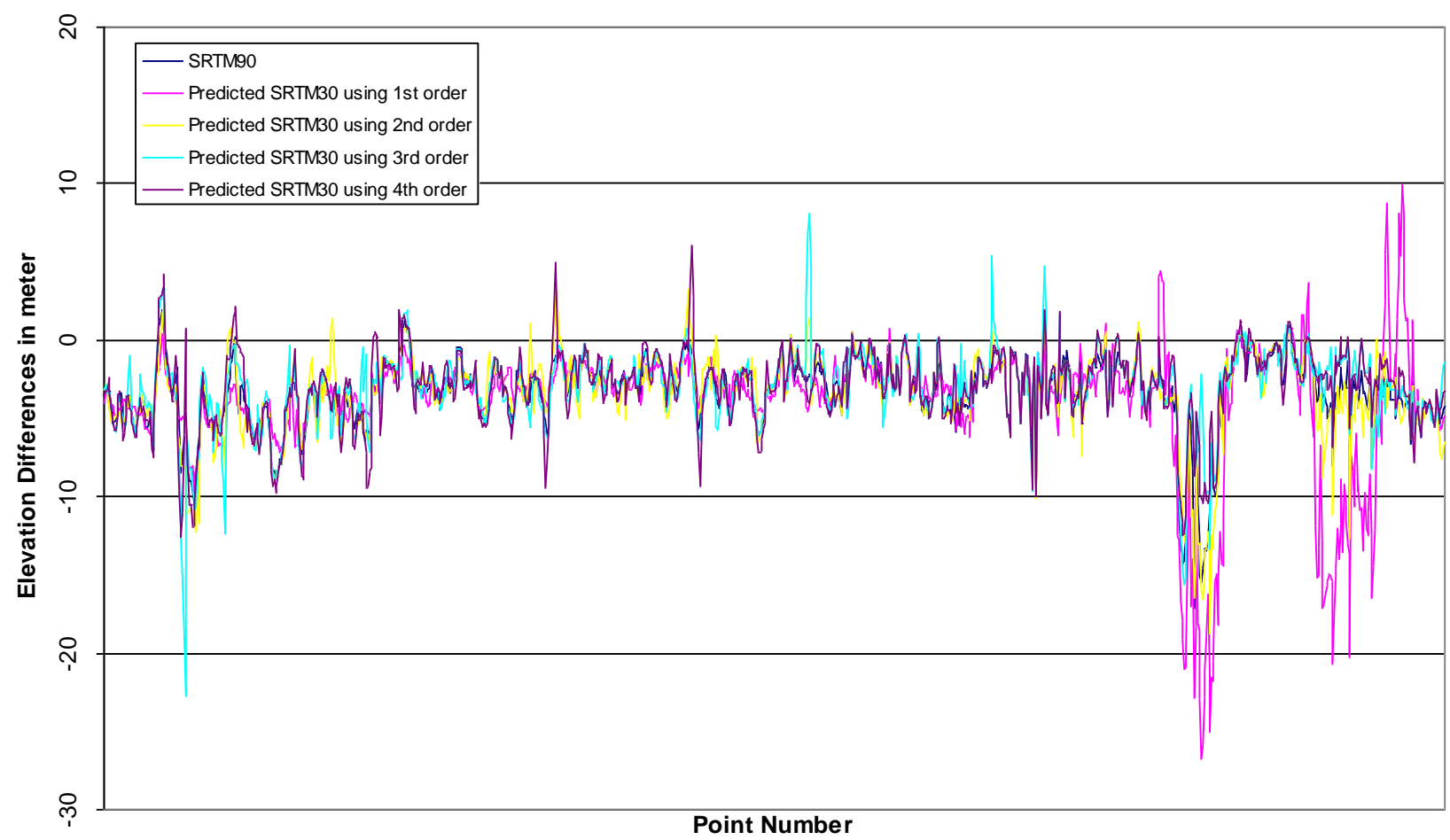

Figure 8: Elevation Differences of the actual ground 1100 points of the study area and SRTM90, and predicted SRTM30

\section{CONCLUSION}

By using the fourth polynomial order, the interpolated SRTM30 was better than other polynomial degrees, specially the third order (bicubic) which was used widely by [9]. Moreover, the two available data of SRTM90 (sampling and averaging) were comparable in terms of elevation differences and statistics. Different performance of the interpolation according to the terrain surface was detected and the SRTM90 can be used as a complete and homogeneous DEM especially in area of flat terrain. In addition it is normally that the editing SRTM90 version_2 is better than the non-editing version. More studies are further warranted to create another interpolation method to improve the results.

Acknowledgment: The author acknowledges NASA, USGS and JPL for providing the SRTM data set. The effort of those who offered reports and sources are greatly appreciated which was helpful and commonly spread by internet.

Note: All data used in this study are freely available from the sources mentioned in the text (see also references)

\section{REFERENCES}

[1] Becek K., (2007) "Comparison of Decimation and Averaging Methods of DER's Resampling" Proceedings of the Map Asia Conference 2007.
[2] David, K., Mark, D. \& Derek, S. (2002) "What's the Point? Interpolation and Extrapolation with a Regular Grid DEM”, Conference ACRS 2002.

[3] Grohmann, C.H., (2006), "Resampling SRTM 3"with Kriging”, GRASS/OSGeo-News 2006 volume 4 December 2006 20-26.

[4] Gorokhovich Y., Voustianiou A., (2006), "Accuracy Assessment of the Processed SRTMbased Elevation Data by GIAR Using Field Data from USA and Thailand and its Relation to the Terrain Characteristics", Remote Sensing of Environment 104 (2006) 409-415.

[5] Gonçalvesa, J. A., Morgadob A. M. (2008), "Use of the SRTM DEM as Georeferencing Tool by Elevation Matching", ISPRS Vol. XXXVII. Part B2. Beijing 2008.

[6] Hermsmeyer D., Guretzki M., Rüffer J., Krüger S., (2008) "Complete SRTM digital Elevation Data for the Arabian Peninsula", Study Carried out by Phoenics GmbH using ArcGIS (Spatial Analyst Module).

[7] Hwang, J, \& Shih, T. (1997) "A Comparison of Bilinear Interpolation, Cubic Convolution, Brownian Interpolation with Least Squares Matching " ,Conference ACRS 1997 
[8] Jarvis A., Rubiano J., Cuero A. (2006), “ Comparison of SRTM derived DEM vs. Topographic Map Derived DEM in the Region of Dapa”, CIAT report 2006.

[9] Keeratikasikon C., Trisirisatayawong I., (2008) "Reconstruction of 30m DEM from 90m SRTM with Bicubic Polynomial Interpolation Method", ISPRS Vol. XXXVII. Part B12. Beijing 2008.

[10]Sachs J., (2001) "Image Resampling", Copyright (c) 2001 Digital Light \& Color.

[11] Mouratids A., Briolep P., Katsamblos K.,(2010 ) “ SRTM 3DEM (versions 1, 2, 3, 4) Validation by Means of Extensive Kinematic GPS measurements: a Case Study from North Greece " ,International Journal of Remote Sensing Vol. 31, No. 23, 10 December 2010, 6205-6222.

[12] Miliaresis G.Ch, Paraschou C.V.E., (2005), "Vertical Accuracy of the SRTM DTED Level 1 of Crete", International Journal of Applied Earth Observation and Geoinformation 7 (2005) 56 4959.
Other Web sites of interest:

[13] NASA/JPL SRTM:

http://www.jpl.nasa.gov/srtm/.

[14] NGA: http://www.nga.mil/

[15] USGS: http://seamless.usgs.gov/.

[16] SRTMFILL:

http://www.3DNature.com/SRTMFILL.html/.

[17] U.S. Geological Survey, EROS Data Center: http://edc.usgs.gov/.

[18] http://dds.cr.usgs.gov/srtm/version2_1/SRTM3/.

[19] http://dds.cr.usgs.gov/srtm/version2_1/Documentat ion/SRTM_Topo.pdf.

[20] http://dds.cr.usgs.gov/srtm/version2_1/SRTM30/sr tm30_documentation.pdf. 